\title{
Gamma Decay of Nuclear Molecular States
}

\author{
F. Haas ${ }^{1}$ \\ ${ }^{1}$ Institut de Recherches Subatomiques, CNRS-IN2P3 et \\ Université Louis Pasteur, F-67037 Strasbourg Cedex 2, France
}

\begin{abstract}
Signatures of deformations and clustering in nuclei can be found in the $\gamma$ decay properties of their excited states. Such signatures are still missing for the 10-called nuclear 'molecular' states which correspond to the resonant states observed in light heavy-ion reactions. Among the new proposal experiments to search for $\gamma$ transitions between highly deformed molecular states, the radiative capture studies looks quite promising.
\end{abstract}

Keywords: gamma decay; deformed, cluster and molecular states $P A C S:$ see : 25.70.ef, $21.10-\mathrm{k}, 24.30 . \mathrm{Cz}, 21.60$. Gx

\section{Introduction}

Strong moment structures in the elastic and reaction channels have been observed in a certain number of light heavy-ion reactions at energies above the Coulomb barrier and up to about $5 \mathrm{MeV}$ per nucleon. For example, these resonant affects are very pronounced in the following collisions between identical bosons: ${ }^{12} \mathrm{C}+{ }^{12} \mathrm{C}$, ${ }^{14} \mathrm{C}+{ }^{14} \mathrm{C},{ }^{16} \mathrm{O}+{ }^{16} \mathrm{O},{ }^{24} \mathrm{Mg}+{ }^{24} \mathrm{Mg}$ and ${ }^{24} \mathrm{Si}+{ }^{24} \mathrm{Si}$. To demonstrate that these resonant structures correspond to highly deformed molecular states in compound nuclei with $\mathrm{A}<60$, the observation of their $\gamma$ decay is essential but unfortunately still missing. The main reason for that is that these states lies generally at high excitation energy, consequently and despite their structure, their $\gamma$-decay widths and orders of magnitude smaller than their total widths $\left(\Gamma_{\gamma} / \Gamma \simeq 10^{-4}-10^{-6}\right)$.

In this report, a few examples will be presented showing the close connections between $\gamma$ decay, nuclear deformation and nuclear clustering. In the case of the molecular states, a status of the experimental structure will be given as well as an overview of experiments underway or planned using the large $\gamma$ arrays and anciallary detectors now available. 


\section{Gamma Decay and Deformation}

Nuclei with highly deformed excited states have been observed during the last twenty years in various regions of the periodic table. The experimental signature of the so-called superdeformed (SD) states is the observation of rotational bands where the SD states are connected by strongly enhanced E2 transitions. In nuclei with A $\geq 60$, SD bands have been observed in the following mass regions: $\mathrm{A}=230-245$, $190,150,130,80$ and 60 . In these nuclei, the physics of the SD wells has been studied in detail through $\gamma$-ray spectroscopy using the most advanced $\gamma$ arrays available (Euroball and Gammasphere). Even higher deformation (hyperdeformation) is predicted by theory but has, up to now, not been seen experimentally at least under the forms of a band of HD states connected by discrete $\gamma$-ray transitions. Recent measurements and calculations indicate that the nucleus ${ }^{126} \mathrm{Be}$ is a good candidate for $\mathrm{HD}$ and a -one month experiment is planned before the mid of the year 2002 to look for HD states in ${ }^{126} \mathrm{Be}$ through the fusion-evaporation reaction ${ }^{64} \mathrm{Ni}\left({ }^{64} \mathrm{Ni}\right.$, $2 \mathrm{n}){ }^{126} \mathrm{Ba}$ at a bombarding energy of $255 \mathrm{MeV}$ using Euroball and the Strasbourg Vivitron accelerator. The main difficulty to populate the HD states is to final the right entrance channel and bombarding energy to reach a 'cold' compound nucleus at an angular momentum high enough to allow feeding of the HD states but alow not too high to survive fission. Similar 'window' effects have been found in the search for molecular resonances and also for superheavy elements.

As said before, strong moment structures have been observed for compound systems with $\mathrm{A}<60$. It is interesting to look for correlations between the resonant phenomena and the recently discovered SD in this mass region. For these lighter nuclei, the most recent and 'textbook' examples of SD is the doubly magic ${ }^{40} \mathrm{Ca}$ nucleus [1]. In this nucleus with a $\mathrm{O}^{+}$spherical ground state, excited bands of levels have been observed built on deformed $0^{+}$states at 3.35 and $5.21 \mathrm{MeV}$ with $4 \mathrm{p}-4 \mathrm{~h}$ and $8 \mathrm{p}-8 \mathrm{~h}$ structures, respectively. The band built on the $0^{+} 8 \mathrm{p}-8 \mathrm{~h}$ state has been estimated up to $\mathrm{J}^{\star}=16^{+}$at $22.06 \mathrm{MeV}$. Through detailed $\gamma$-ray spectroscopy, it has been shown that this band is SD with a large prolate deformation of $\beta=$ 0.59. It should be noticed that due to the high efficiency of the $\gamma$-array (in this case Gammasphere), the $\gamma$-decay of these very deformed states could be observed up to an excitation energy of $22 \mathrm{MeV}$ which for ${ }^{40} \mathrm{Ca}$ is about $15 \mathrm{MeV}$ above the ${ }^{36} \mathrm{Ar}+\alpha$ threshold energy. This is of course very good news for our search of $\gamma$ decay from molecular states which are generally located at high excitation energies. The nucleus ${ }^{36} \mathrm{Ar}$ is another example of light machine where, an SD band has been observed [2]. It is also a good candidate to look for eventual links between the SD band and a known molecular band (MB) composed of resonances seen in ${ }^{16} \mathrm{O}+$ ${ }^{20} \mathrm{Ne}$ and ${ }^{12} \mathrm{C}+{ }^{20} \mathrm{Mg}$ reactions. The moment of inertia of $\mathrm{MB}$ is larger than that of SD but up to the highest known spin state in the SD but up to the highest known spin state in the $\mathrm{SD}$ band $\left(\mathrm{J}^{H}=16^{+}\right.$at $\left.\mathrm{E}_{x}=22.36 \mathrm{MeV}\right)$, the difference in energy between the MB and SD sttes is still of $\sim 20 \mathrm{MeV}$ which makes the observation of $\gamma$ transitions between these bands extremely difficult at least in the considered angular momentum range. Another s-d shell nucleus under active study is ${ }^{82} \mathrm{~S}$, for which SD 
and molecular ${ }^{16} \mathrm{O}-{ }^{16} \mathrm{O}$ bands are predicted by various modele but have, up to now, not be discovered. But the serach is actually continuing using fusion-evaporation, transfer and radiative capture reactions to do a detailed $\gamma$ spectroscopy of ${ }^{32} \mathrm{~S}$ at excitation energies above $8 \mathrm{MeV}$. As we have learned recently, large deformations can be found in very neutron rich light nuclei. The case of ${ }^{34} \mathrm{Mg}$ with $\mathrm{N}=22$ is probably the best example [3]. From a Coulomb excitation and $\gamma$ decay experiment, the E2 strength of the $2^{+} \rightarrow 0^{+24} \mathrm{Mg}$ transition has been scanned and corresponds to a large prolate deformation of $\beta=0.58$. Of course other members of this eventual ground state band have to be observed before we can speak of a SD ground state band, but it is interesting to note that the strength of the ${ }^{34} \mathrm{Mg} \mathrm{E} 2\left(2^{+} \rightarrow 0^{+}\right)$teamsiton(19W.u.) is comparable with the strength of the ${ }^{16} \mathrm{O} \mathrm{E} 2\left(2^{+} \rightarrow 0^{+}\right)$transition (27 W.u.) between the $4 \mathrm{p}-4 \mathrm{~h} 2^{+}$and $0^{+} \alpha$ cluster states. This speaks not only in favour of a large deformation for ${ }^{34} \mathrm{Mg}$ but also for an sup- cluster structure of this very neutron rich nucleus.

\section{Gamma Decay and Clustering}

Alpha cluster states are well known in light nuclei at the beginning of the s-d and $f-p$ shells. From this characteristic $\gamma$ decay, they have been gouped into bands for which good examples exist in ${ }^{16} \mathrm{O}\left({ }^{12} \mathrm{C}+\alpha\right),{ }^{18} \mathrm{O}\left({ }^{14} \mathrm{C}+\alpha\right),{ }^{20} \mathrm{Ne}\left({ }^{16} \mathrm{O}+\alpha\right)$ and ${ }^{44} \mathrm{Ti}\left({ }^{40} \mathrm{Ca}\right.$ $+\alpha)$. The ${ }^{18} \mathrm{O}$ nucleus is a particularly good example because the cluster states are not only connected by $\mathrm{H} 1$ or $\mathrm{E} 2$ transitions but also by strong $\mathrm{E} 1$ transitions which are signatures of transitions between $\alpha$ cluster (peer shape-like) configurations and are not forbidden like in $\mathrm{N}=\mathrm{Z}$ nuclei. In light-nuclei, the best examples of cluster states are: the ground state of ${ }^{8} \mathrm{Be}$ with an $\alpha-\alpha$ structure; the $3 / 2^{-}$and $5 / 2^{-}$states of ${ }^{9} \mathrm{Be}$ at $\mathrm{E}_{x}=0.0$ and $2.43 \mathrm{MeV}$ with an $\mathrm{n}-\alpha-\alpha$ and an enhanced $\mathrm{E} 2$ transition of 24 W.m. between them; the ${ }^{10} \mathrm{Be} 0^{+}$state at $\mathrm{E}_{x}=6.18 \mathrm{MeV}$ and $2.43 \mathrm{MeV}$ with an $2 \mathrm{n}-\alpha-\alpha$ structure $\left(\right.$ no $\mathrm{O}^{+}$state at $\mathrm{E}_{x}=7.65 \mathrm{MeV}$ with a $\alpha-\alpha-\alpha$ structure which decays into ${ }^{8} \mathrm{Be}+\alpha$ but also by an E2 transition of $8 \mathrm{~W}$.u. to the $2^{+}$state even so this state is much less deformed; the ${ }^{16} \mathrm{O} 0^{+}, 2^{+}$and $4^{+}$state at $\mathrm{E}_{x}=$ 6.05, 6.92 and 10.36 MeV which are numbers of a ${ }^{12} \mathrm{C}+\alpha(4 \mathrm{p}-4 \mathrm{~h})$ cluster band and which $\gamma$-decay by two strong intruband E2 transitions of 27 and $65 \mathrm{~W} . u$. In this field of a clustering and $\gamma$ decay, new experiments proposed by the Berlin group are underway to search for an $\gamma$-decays in light neutron rich $\mathrm{Be}$ and $\mathrm{C}$ isotopes with $x n-\alpha-\alpha$ and $x n-\alpha-\alpha-\alpha$ structures $[4,5]$. In this case the $\mathrm{Be}$ and $\mathrm{C}$ isotope are populated through transfer reactions induced by ${ }^{18} \mathrm{O}$ on ${ }^{9} \mathrm{Be}$, they are detected as binary reaction fragments in coincidence with $\gamma$-rays observed in the Euroball array, the high efficiency of which is neutral to observe these week branches with expected $\Gamma_{\gamma} / \Gamma$ ratios less than $10^{-3}$. 


\section{Gamma Decay, Resonant Structures and Molecular States}

The question of whether the resonant structures seen in certain weak absorption light heavy-ions reactions are scattering entrance channel effects or genuine high excitation strongly deformed molecular states will only be definitively settled if the $\gamma$-decay of such resonances can be observed. The ${ }^{12} \mathrm{C}+{ }^{12} \mathrm{C}$ reaction is the best studied collision in Nuclear Physics, very detailed excited functions have been measured for the elastic and main reaction channels at bombarding energies between 1 and $10 \mathrm{MeV}$ per nucleon. Pronounced resonant structure exist in this system from the Coulomb barrier to $5 \mathrm{MeV}$ per nucleon and if decay $\gamma$-rays have to be discovered it will probably be first in ${ }^{12} \mathrm{C}+{ }^{12} \mathrm{C}$ where the resonant effects are much stronger than in any other system.

This reaction has thus been used in several experiment aimed to search for the $\gamma$-decay of resonant structures. The experimental method adapted was to choose a bombarding energy corresponding to a resonance in the elastic scattering channel and to search for eventual $\gamma$ rays in competition with the corresponding binary fragment exit channel. The first experiments were performed at relatively high energy $\left(\mathrm{E}_{C M}=26 \mathrm{MeV}\right)$ and all the reported results were negative (see [6] and references therein). This is in fact not so surprising because at these energies the number of open channels is large and also the resonance itself has a complex cluster structure, the resonant flux is thus split among a large number of channels and configurations. To search for small branghings, it is always adisable to go to lower energies and smaller phase-space. A study was thus reported by the Strasbourg group [6] at an energy of $\mathrm{E}_{C M}=16.45 \mathrm{MeV}$ which corresponds to a well known and isolated $10^{+}$resonance in the elastic ${ }^{12} \mathrm{C}+{ }^{12} \mathrm{C}$ scattering. The $\gamma$ rays were detected in the highly efficient 4 'Château de Cristal' array composed of $\mathrm{BaF}_{2}$ detectors. After are full week of beamtime 7 'good' events were recorded with a mean energy of $\mathrm{E}_{\gamma}=6.9 \mathrm{MeV}$ which could correspond to $\mathrm{E} 2$ transitions between the well known $10^{+}$and $8^{+12} \mathrm{C}+{ }^{12} \mathrm{C}$ resonant states. The deduced branching ratio $\frac{\Gamma_{\gamma}}{\Gamma}=(1.2 \pm 0.4) \times 10^{-5}$ is quite large and it is evident that such a result needs confirmation from a much larger scale experiment for which all the required and crucial experimental conditions concerning beam, $\gamma$ and fragment detectors could not be satisfied at the same time up to now.

At the beginning of the rightim, appeared in the literature the first results concerning the heavy-ion radiative caption reaction ${ }^{12} \mathrm{C}\left({ }^{12} \mathrm{C}, \gamma\right){ }^{24} \mathrm{Mg}$ [7] at energies between $\mathrm{E}_{C M}=5$ and $11 \mathrm{MeV}$. High energy giant-resonance $\gamma$-ray decays to the low-lying states in $\left.{ }^{24} \mathrm{Mg}\left(0^{+}\right), 2^{+}, 4^{+}\right)$were observed using a large NaI detector. Pronounced and correlated resonant structure was found in all the channels. However with such a set-up, it was not possible to look for multi-step decays through lowlying states in ${ }^{24} \mathrm{Mg}$. It was therefore nuclear whether multi-step decays existed or how preponderant they might be relative to the observed single step decays. Moreover, multi-step decays might opulate exotic and non-yrast states not usually seen in heavy-ion reactions; such states being selectively populated by their relation to 
the structure of the capture resonant states.

To look for these multi-step decays, experiments were performed at Argonne and Berkeley using Gammasphere, - a highly segmented $4 \pi \gamma$ array [8]. The radiative capture ${ }^{12} \mathrm{C}+{ }^{12} \mathrm{C}$ experiment was investigated at a beam energy of $\mathrm{E}_{C M} \sim 8 \mathrm{MeV}$ $[9,10]$ close to a $\mathrm{J}^{\pi}=4^{+}$resonance reported in Ref. [7]. The $\gamma$-detector was used as a calorimeter -and a clear unel-point was seen in the sum-energy spectrum corresponding closely to the maximum energy expected from a radiative capture process. By gating on this fully captured events, it was possible to deconvoluate the $\gamma$ cascade which surprisingly enough shows selective feedings of levels belonging to various ${ }^{24} \mathrm{Mg}$ bands, in particular the $\mathrm{K}^{\pi}=2^{+}$band and its $4^{+}$number at $\mathrm{E}_{x}=$ $6.01 \mathrm{MeV}$. An even more interesting obsevation is the strong feedings of states in ${ }^{24} \mathrm{Mg}$ at energies around $10 \mathrm{MeV}$ and of unknown structure. These very preliminary and still poor statistics results [8-10] seems to be in qualitative agreements with a decay scenario of radiative capture states proposed by Baye and Descouvements $[11,12]$ in the frameworks of a microscopic study of the ${ }^{12} \mathrm{C}+{ }^{12} \mathrm{C}$ system with the Coordinate Generator Method. They showed that the ${ }^{12} \mathrm{C}-{ }^{12} \mathrm{C}$ molecular structure (MB) and a band of broad barrier resonance (BB). Considering the structure of these bands, a favored decay of levels of $\mathrm{BB}$ to levels of $\mathrm{MB}$ is expected. In other words, the number of the molecular band (MB) are the downway states of the broad barrier resonance of $\mathrm{BB}$.

An experiment is underway to measure with the Argonne Fragment Mass Analyser (FMA) the total radiative capture cross section. From previous measurements $[8,9]$, it is estimated that the multi-step process is at least two times stronger than the single-step giant resonance decays. In a future experiment, it is planned to use FMA with Gammasphere, to measure with good statistics the different pathways of the multi-step $\gamma$ decays and to identify in the 10 to $12 \mathrm{MeV}$ excitation energy region the downway states which correspond probably to ${ }^{24} \mathrm{Mg}$ states having a pronounced ${ }^{12} \mathrm{C}-{ }^{12} \mathrm{C}$ molecular structure. Such radiative capture studies could be estimated to neighbouring systeme, where resonant structure has been observed, like ${ }^{12} \mathrm{C}+{ }^{16} \mathrm{O}$, ${ }^{12} \mathrm{C}+{ }^{14} \mathrm{C}$ and ${ }^{16} \mathrm{O}+{ }^{16} \mathrm{O}$ to search for molecular configurations and their $\gamma$ decay in ${ }^{28} \mathrm{Si},{ }^{26} \mathrm{Mg}$ and ${ }^{32} \mathrm{~S}$, respectively.

Concerning the $\gamma$-decay of molecular states in ${ }^{24} \mathrm{Mg}$, it is - a low proposed to investigate the ${ }^{24} \mathrm{Mg}$ on ${ }^{12} \mathrm{C}$ reaction at the Strasbourg Vivitron accelerator using Euroball and the Berlin Binary Recoil Spectrometer. The goal of this experiment (complementary to the radiative capture study) is to measure the $\gamma$ branching ratios of unbound, low spin, deformed cluster ${ }^{24} \mathrm{Mg}$ states in, an excitation energy region between 9 and $14 \mathrm{MeV}$ which are the threshold energies of the ${ }^{20} \mathrm{Ne}+\alpha$ and ${ }^{12} \mathrm{C}$ $+{ }^{12} \mathrm{C}$ decay channels respectively.

Very strong resonant structure has been observed in the elastic and inelastic channels of the ${ }^{24} \mathrm{Mg}+{ }^{24} \mathrm{Mg}$ reaction. Cluster model calculations [13] predicts an unusually strong decay branching ratio $\left(\Gamma_{\gamma} / \Gamma \simeq 10^{-4}\right)$ for $\gamma$ transitions between resonant sstates. Such predictions for such $\gamma$ transitions using the highly efficient $\gamma$ arrays actually available or under development. What the correlations between resonances and superdeformation is concerned, a good case to study would be the 
feeding of the SD band in ${ }^{40} \mathrm{Ca}$ through the $2 \alpha$ or ${ }^{8} \mathrm{Be}$ evaporation channels of the ${ }^{24} \mathrm{Mg}+{ }^{24} \mathrm{Mg}$ reaction at ' $\mathrm{ns}$ ' an 'off' resonance energies. This should allow to establish a direct link through $\alpha$ decay between the resonant and molecular states in ${ }^{48} \mathrm{Cr}$ and the $\mathrm{SD}$ states in ${ }^{40} \mathrm{Ca}$.

\section{Conclusions}

Different experiments are actually underway or planned to search for $\gamma$ rays between deformed cluster states or highly deformed molecular states in light nuclei with A $<60$. These experiments are extremely difficult because of the very small $\Gamma_{\gamma} / \Gamma$ branching ratios, they are very demanding and require not only large amounts of beam time but also the combinations of high efficiency fragment and $\gamma$-ray detection arrays. In the nuclear molecular saga, the most spectacular results have always been obtained for the ${ }^{12} \mathrm{C}+{ }^{12} \mathrm{C}$ reaction. Again today, the most promising experiment to search for $\gamma$ rays between molecular states is the resonant radiative capture ${ }^{12} \mathrm{C}+$ ${ }^{12} \mathrm{C}$ reactions in which the populated ${ }^{24} \mathrm{Mg}$ 'linking' ${ }^{12} \mathrm{C}-{ }^{12} \mathrm{C}$ states. If successfull, such radiative capture experiments could of course be extended to neighbouring resonant systems like ${ }^{12} \mathrm{C}+{ }^{16} \mathrm{O},{ }^{12} \mathrm{C}+{ }^{14} \mathrm{C}$ and ${ }^{16} \mathrm{O}+{ }^{16} \mathrm{O}$.

\section{References}

1. E.Ideguchi et al., Phys. Rev. Lett. 67 (2001) 222501

2. C.E. Svenson et al., Phys. Rev. C63 (2001) 061301.

3. H. Iwasahi et al., Phys. Lett. 522B (2001) 227.

4. W. Von Oertzen, Z. Phys. A354 (1996) 37 and Z. Phys. A257 (1997) 355.

5. M. Milin and W. von Oertzen Eur. Phys. J. A14 (2002) 295

6. F. Haas et al., Nuovo Cimento 110 A (1997) 989.

7. A.M. Nathan, A.M. Sandorfi and T.J.Bowles, Phys. Rev. C24 (1981) 932.

8. C.J. Lister et al., Physics Division Annual Report 2001, Argonne National Lab., ANL-02/15 (2002), p. 45.

9. D.G. Jenkins et al., Proceedings of the Symposium on Cluster Aspects of Quantum Many Body Systems, eds. kato et al., Kyoto, 2001; to be published by World Scientific.

10. D.G. Jenkins (private communication).

11. D. Baye and P. Descouvement, Nucl. Phys. A419 (1984) 397.

12. P. Descouvement, and D. Baye, Phys. Lett. 196B (1986) 143.

13. E. Megaki, Prog. Theo. Phys. Suppl. No 132 (1998) 135. 